\title{
Problèmes globaux dans le théorie des équations intégrales de Volterra.
}

\author{
par C. Conduneanu (Iasi, Romania)
}

\begin{abstract}
Résumé. - Le but du travail est l'étude du comportement global des solutions des équations intégrales non-linéxires de Volterra. On utilise les théorèmes de point fixe et d'autres moyens de l'Analyse fonctionnelle.
\end{abstract}

Dans ce travail, nous allons considérer quelques problèmes globaux concernant les équations intégrales vectorielles de la forme

$$
x(t)=h(t)+\int_{0}^{t} k(t, s) f(s, x(s)) d s .
$$

Il s'agit d'établir l'existence des solutions de l'équation (E) dans certains espaces fonctionnels, en suivant la méthode développée par J. L. Massera et J. J. SGHÄFFer [8], [9] pour les systèmes d'équations différentielles ordinaires. L'appartenance même de la solution à tel ou tel espace fonctionnel, lui confère un certain genre de comportement global.

1. - L'espace fonctionnel fondamental pour nos considérations sera l'espace $C_{\mathfrak{c}}\left(R_{+}, R^{n}\right)$ des fonctions vectorielles continues sur le demi-axe $R_{+}=\{t ; t \geq 0\}$, à valeurs dans l'espace euclidien $n$-dimensionel $R^{n}$, doué de la topologie de la convergence uniforme sur tout compact de $R_{+}$. Pour les détails concernant les propriétés de cet espace, nous renvoyons le lecteur au livre de N. Bourbaki [3]. Mentionnons seulement que l'espace $C_{\mathrm{c}}\left(R_{+}, R^{n}\right)$ est un espace vectoriel topologique, localement convexe et métrisable, fait qui permet l'utilisation des suites dénombrales.

Nous allons considérer aussi d'autres espaces fonctionnels, surtout des espaces de BANACH de fonctions continues sur $R_{+}$, dont la topologie est plus forte que la topologie de l'espace $C_{c}\left(R_{++}, R^{n}\right)$. Un exemple de tel espace est celui des fonctions continues et bornées sur $R_{+}$, à valeurs dans $R^{n}$, la norme étant définie par

$$
|x(t)|=\sup _{t \geq 0}\|x(t)\|
$$

où $\|x(t)\|$ désigne la longueur euclidienne du vecteur $x(t)$. La topologie de cet espace est évidemment la topologie de la convergence uniforme sur $R_{+}$. Nous allons désigner cet espace par $C$, au lieu de $C\left(R_{+}, R^{n}\right)$, pour simplifier l'écriture. 
Dans ce qui suit, nous allons considérer aussi les espaces $O_{g}=C_{g}\left(R_{+}, R^{n}\right)$ des fonctions continues sur $R_{+}$, à valeur dans $R^{n}$, telles que

$$
\|f(t)\| \leq A_{f} g(t), \quad t \geq 0
$$

où $g(t)$ est une fonction réelle, continue et positive sur $R_{+}$. $A_{f}$ est une constante positive qui dépend de $f$.

2. - Soit maintenant $k(t, s)$ une matrice du type $n \times n$, dont les éléments sont des fonctions réelles, continues pour $0 \leq s \leq t<+\infty$.

Considérons sur $C_{\mathfrak{e}}\left(R_{+}, R^{n}\right)$ l'opérateur de VolterRa

$$
(T f)(t)=\int_{0}^{t} k(t, s) f(s) d s .
$$

$T$ est évidemment linéaire et continu sur $O_{e}\left(R_{+}, R^{n}\right)$.

Soient $B=B\left(R_{+}, R^{n}\right), \quad D=D\left(R_{+}, R^{n}\right)$ deux espaces de BaNaCH de fonctions continues. Donc $B, D \subset O_{c}\left(R_{+}, R^{n}\right)$.

Nous dirons que la paire $(B, D)$ est admissible par rapport à l'opératenr $T$, si l'on a $T B \subset D$.

Le résultat fondamental pour ce qui suit peut s'énoncer de la manière suivante :

Lemme. - Soient $B$ et $D$ deux espaces de Banach de fonctions continues dont les topologies sont plus fortes que la topologie de $C_{c}\left(R_{+}, R^{n}\right)$. Supposons que la paire $(B, D)$ est admissible par rapport à l'opérateur $T$. Alors $T$ est continu sur $B$, c'est-à-dire

$$
|T f|_{D} \leq K|f|_{B}, \quad f \in B
$$

$K$ étant une constante positive.

Démonstration. - Nous allons montrer que l'opérateur $T$ est fermé. La continuité sera la conséquence du théorème du graphique fermé (voir p. ex. [7]). Admettons done qu'on a $f_{n} \rightarrow f$ dans $B$ et $T f_{n} \rightarrow g$ dans $D$. Vu que $T$ est continu sur $C_{c}\left(R_{+}, R^{n}\right)$ et que $f_{m} \rightarrow f$ dans $B$, done à plus forte raison dans $C_{c}\left(R_{+}, R^{n}\right)$, on aura $T f_{n} \rightarrow T f$ dans $C_{c}\left(R_{+}, R^{n}\right)$. D'autre part, $T f_{n} \rightarrow g$ dans $D$ a pour eonséquence $T f_{n} \rightarrow g$ dans $C_{e}\left(R_{+}, R^{n}\right)$. L'espace $C_{c}\left(R_{+}, R^{n}\right)$ étant séparé, on aura $T f=g$, ce qui démontre le fait que le graphique de l'opé. rateur $T$ est fermé (dans $B \times D$ ).

Remarque. - La constante $K$ qui figure dans la relation (3) dépend évidemment de $k(t, s), B$ et $D$. 
C. Conduneanu: Problèmes globautx dans le théorie des équations, etc. 351

3. - Maintenant, on peut établir aisément un théorème d'existence et unicité de la solution de l'équation (E), appartenant à un espace fonetionnel de BANACH $D$, dont la topologie est plus forte que la topologie de l'espace $C_{e}\left(R_{+}, R^{n}\right)$.

Thíont̀me 1. - Considérons l'équation (E) et admeltons les conditions suivantes:

a) $B$ et $D$ sont des espaces de Banach plus forts que $C_{o}\left(R_{+}, R^{n}\right)$, tels $q u e(B, D)$ est admissible par rapport à l'opérateur $T$, défini par (2) ;

b) $x(t) \rightarrow f(t, x(t))$ est un opérateur défini sur l'ensemble $S=\{x(t)$; $x(t) \in D,|x| D \leq \rho\}$, dont les valeurs appartiennent $\dot{a} B$, tel que

$$
|f(t, x(t))-f(t, y(t))|_{B} \leq \lambda|x(t)-y(t)|_{D}, x(t), y(t) \in S,
$$

où $\lambda$ est une constante positive;

c) $h(\mathrm{t}) \in D$.

Alors il existe une solution unique $x(t) \in S \subset D$ de l'équation $(E)$, dès que $|h(t)|_{D}, \lambda$ et $|f(t, 0)|_{B}$ sont assez petits.

Démonstration. - On utilisera le théorème du point fixe de BanacH, en choisissant $D$ comme espace fondamental.

Définissons sur $S$ un opératenr $x \rightarrow U x$ de la manière suivante:

$$
\left(U_{x)}(t)=h(t)+\int_{0}^{t} k(t, s) f(s, x(s)) d s .\right.
$$

Pour simplifier, nous écrirons $U x(t)$ au lien de $(U x)(t)$.

On aura évidemment

$$
|U x(t)-U y(t)|_{D} \leq K|f(t, x(t))-f(t, y(t))|_{B},
$$

en tenant compte du lemme plus haut établi. L'inégalité (4) de l'énoncé du théorème 1 nous permet d'écrire

$$
|U x(t)-U y(t)|_{D} \leq K \lambda|x(t)-y(t)|_{D}
$$

quels que soient $x(t), y(t) \in S$. Par conséquent, si l'on admet

$$
\lambda<K^{-1}
$$

l'opératear $U$ est un opérateur de contraction. 
Il faut s'assurer encore du fait que $U S \subset S$. Il est évident qu' on a

$$
|U x|_{D} \leq|h(t)|_{D}+K|f(t, x(t))|_{B} .
$$

Mais

$$
\begin{gathered}
|f(t, x(t))|_{B} \leq|f(t, x(t))-f(t, 0)|_{B}+|f(t, 0)|_{B} \leq \lambda|x(t)|_{D}+|f(t, 0)|_{B} \leq \\
\leq \lambda_{P}+|f(t, 0)|_{B} .
\end{gathered}
$$

Done, il suffit d'admettre

$$
|h(t)|_{D}+\lambda K_{\rho}+K|f(t, 0)|_{B} \leq \rho,
$$

pour assurer l'inclusion $U S \subset S$.

Si l'on tient compte de la condition (7), on obtient

$$
|h(t)| p+K|f(t, 0)|_{B} \leq p(1-\lambda K),
$$

inégalité qui est satisfaite pour $|h(t)|_{D}$ et $|f(t, 0)|_{B}$ assez petits.

Remarque 1. - On peut évidemment admettre que $x(t) \rightarrow f(t, x(t))$ est défini sur $D$ tout entier. Alors on peut renoncer anx conditions de limitation pour $h(t)$ et $f(t, 0)$, en gardant sealement la condition de contraction (7). Il en résulte l'existence et l'unicité de la solution de l'équation (E) dans l'espace $D$.

Remarque 2. - Il est aisé de voir que le résultat plus haut établi reste valable quand on remplace l' espace enclidien $R^{n}$ par un espace de BANAcH $F$. Dans ce dernier cas, $k(t, s)$ aura des valeurs dans l'espace des opérateurs linéaires bornós de $F$.

Remarque 3. - Un résultat analogue an précédent, concernant les systèmes différentiels, a été établi dans [4].

4. - Nous allons démontrer maintenant un autre théorème, semblable au précédent, concernant seulement l'existence de la solution de l'équation $(E)$.

THF́onìme 2. - Considérons l' équation (E) et admettons les conditions suivantes :

a) $B$ et $D$ sont des espaces de Banach plus forts que $C_{c}\left(R_{+}, R^{n}\right)$, tels $q u e(B, D)$ est admissible par rapport à l'opérateur $T$; 
C. Corduneanu: Problèmes globaux dans le théorie des équations, etc. 353

b) $x(t) \rightarrow f(t, x(t))$ est un opérateur continu sur la fermeture $\bar{S}$ dans $C_{d}\left(R_{+}, k^{n}\right)$ de l'ensemble $S=\left\{x(t) ; x(t) \in D,|x|_{D} \leq \rho\right\}$, à valeurs dans $B$, tel que

$$
\begin{array}{ll}
|f(t, x(t))|_{B} \leq r, & x(t) \in \bar{S} \\
\|f(t, x(t))\| \leq \lambda(t), \quad t \geq 0, & x(t) \in \bar{S}
\end{array}
$$

où $r>0$ et $\lambda(t)$ est une fonction continue sur $R_{+}$;

c) $h(t) \in D$.

Alors, il existe au moins une solution $x(t) \in S \subset D$ de l'équation $(E)$, dès que $|h(t)|_{D}$ et $r$ sont assez petits.

Démonstration - Cette fois on utilisera le principe du point fixe de Sohadder et Trohoroff. L'espace fondamental sera l'espace $C_{c}\left(R_{+}, R^{n}\right)$, bien que la solution appartiendra à l'espace $D$.

Sur l'ensemble $\bar{S} \subset C_{c}\left(R_{+}, R^{n}\right)$, qui est évidemment convexe (étant la fermeture d'un ensemble convexe), définissons l'opérateur $x \rightarrow U x$ par la formule (5), c'est-à-dire

$$
U x(t)=h(t)+\int_{0}^{t} k(t, s) f(s, x(s)) d s .
$$

Les valeurs de cet opérateur appartiennent à l'espace $D$, fait qui suit immédiatement des conditions admises. On aura pour $x(t) \in \bar{S}$

$$
|U x(t)|_{D} \leq|h(t)|_{D}+K r .
$$

Si l'on admet la condition

$$
|h(t)| D+K r \leq p
$$

il en résulte

$$
U \bar{S} \subset S \subset \bar{S}
$$

ce qui sera nécessaire pour l'application du théorème de point fixe.

Remarquons encore que l'opérateur $U$ est continu. En effet, $x(t) \rightarrow f(t, x(t))$ est continu de $C_{c}\left(R_{+}, R^{n}\right)$ à $B$ et $f(t) \rightarrow \int_{0}^{t} k(t, s) f(s) d s$ est continu de $B$ à $D$. Done 


$$
x(t) \rightarrow \int_{0}^{t} k(t, s) f(s, x(s)) d s
$$

est continu de $C_{\mathrm{c}}\left(R_{+}, R^{n}\right)$ à $D$. $\grave{\Lambda}$ plus forte raison, cet opérateur sera continu de $C_{c}\left(R_{+}, R^{n}\right)$ à $C_{c}\left(R_{+}, R^{n}\right)$, ce qui démontre la continuité de $U$.

Pour l'application du théorème de SoHadder et TrchonofF, il reste à montrer l'existence d'un ensemble compact dans $C_{c}\left(R_{+}, R^{n}\right)$, soit $A$, tel que $U \bar{S} \subset A \subset \bar{S}$. Nous allons établir que les fonctions appartenant à $U \bar{S}$ sont uniformément bornées et équicontinues sur tout intervalle fini de $R_{+}$. On pourra prendre alors pour $A$ la fermeture de l'ensemble $U \bar{S}$ dans $C_{\mathrm{c}}\left(R_{+}, R^{n}\right)$.

On obtient sans difficulté

$$
\|U x(t)\| \leq\|h(t)\|+\int_{0}^{t}\|k(t, s)\| \lambda(s) d s,
$$

ce qui démontre que les fonctions appartenant à $\overline{U S}$ sont uniformément bornées sur tout intervalle fini de $R_{+}$, le second membre de (16) étant une fonction continue sur $R_{+}$. On a

$$
\begin{gathered}
U x(t)-U x(\tau)=h(t)-h(\tau)+ \\
+\int_{0}^{t}\{k(t, s)-h(\tau, s)\} f(s, x(s)) d s+\int_{\tau}^{t} k(\tau, s) f(s, x(s)) d s .
\end{gathered}
$$

Soit maintenant $a>0$ un nombre donné et posons $\lambda_{a}=\sup \lambda(t), k_{a}=\sup$ $\| k(t, s \|, 0 \leq s \leq t \leq a$. Admettons maintenant $0 \leq \tau, t \leq a$. On obtient

$$
\begin{gathered}
\|U x(t)-U x(\tau)\| \leq\|h(t)-h(\tau)\|+ \\
+\lambda_{a} \int_{0}^{a}\|k(t, s)-k(\tau, s)\| d s+k_{a} \lambda_{a}|t-\tau| .
\end{gathered}
$$

La continuité uniforme de $h(t)$ sur $[0, a]$ et de $k(t, s)$ sur $0 \leq s \leq t \leq a$ assurent, compte tenant de (17), l'équicontinaité des fonctions de l'ensemble $U \bar{S}$.

Les conditions dn théorème de SOHAUDkR et TxchoNofr étant satisfaites, il s'ensuit l'existence d'un élément fixe (au moins) de l'opérateur $U$, ce qui démontre le théorème 2.

Remarque. - Un résultat analogue pour les systèmes différentiels est dû à l'auteur 4] et à Pr. Hartman et N. ONUChic [6]. 
C. Cordunnanu: Problèmes globaus dans te théorie des équations, etc. 355

5. - Nous allons considerer dans ce qui suit les espaces $C_{g}\left(R_{+}, R^{n}\right) q u$ nous avons mentionnés plus haut.

Supposons done que $g(t)$ est une fonction reélle, continue et positive sur $R_{+}$. Pour organiser l'ensemble des fonctions appartenant à $C_{\mathrm{e}}\left(R_{+}, R^{n}\right)$ ef satisfaisant à la condition (1) comme espace de BANACH, dont la topologie est plus forte que la topologie de $O_{c}\left(R_{+}, R^{n}\right)$ on définit la norme à l'aide de la relation

$$
|f(t)|_{g}=\sup \frac{\|f(t)\|}{g(t)}, \quad t \in R_{+} .
$$

Il est aisé de voir que l'ensemble des fonctions satisfaisant à la condition (1) devient ainsi un espace de BANAor. De plus, la convergence dans $C_{g}$ entraîne la convergence uniforme sur tout intervalle fini de $R_{-+}$. En effet, si $a>0$, on aura

$$
\sup _{0 \leq t \leq a}\|f(t)\| \leq|f(t)|_{g} \cdot \sup _{0 \leq t \leq a} g(t)
$$

Pour $g(t) \equiv 1$ on obtient l'espace des fonctions continues bornées sur $R_{+}$; c' est-à-dire $C_{g}\left(R_{+}, R^{n}\right)=C$, pour $g(t) \equiv 1$. Pour $g(t)=\exp \{\alpha t\}, \alpha$ étant un nombre réel, on obtient une classe d'espaces qui ont été récemment étudiés [5], en relation avec la théorie des équations intégrales.

Il faut remarquer que les espaces $C_{g}$, pour $g(t)=\exp \left\{\int_{0}^{t} \lambda(s) d s\right\}$, ont eté considérés par A. Breleokr [2] qui a montré leur utilié dans l'étude des problèmes globaux concernant les équations fonctionnelles.

Une question des plus importantes concernant l'applicabitité des théorèmès 1 et 2 est de savoir quand la paire $\left(C_{g}, C_{G}\right)$ est-elle admissible par rapport à l'opérateur $T$. On peut donner une réponse complète à cette question.

THक́onème 3. - La paire $\left(C_{g}, C_{G}\right)$ est admissible par rapport à l'opérateur $T$ défini par (2), si et seulement si

$$
\int_{0}^{t}\|k(t, s)\| g(s) d s \leq A G(t), \quad t \geq 0
$$

oì $A$ est une constante positive.

Démonstration. La suffisance de la condition (19) est évidente pour avoir $T C_{g} \subset C_{G}$. Il reste à montrer la nécessité de cette condition. Nous allons supposer, en premier lieu, qu' on a $n=1$. Puis, le cas $n>1$ sera réduit au cas précédent. 
C. Conduneanu: Problèmes globaux dans le théorie des équations, etc.

En supposant que la condition (19) n'est pas satisfaite, nous allons montrer que l'inclusion $T C_{g} \subset C_{G}$ n'est pas possible.

Admettons done que la condition (19) n'ait pas lieu. On peut trouver alors une suite $\left\{t_{m}\right\}$ de nombres positifs, tels que

$$
\int_{0}^{t_{m}}\left|k\left(t_{m}, s\right)\right| g(s) d s>m G\left(t_{m}\right), \quad m \geq 1
$$

(La suite $\left\{t_{m}\right\}$ est nécessairement non bornée). Soit maintenant $m \geq 1$ fixé. Posons

$$
\varphi_{m}(t)=g(t) \operatorname{sign} k\left(t_{m}, t\right), \quad 0 \leq t \leq t_{m} .
$$

La fonction $\varphi_{m}(t)$ est mesurable (étant le produit de deux fonctions mesurables) sur l'intervalle $0 \leq t \leq t_{m}$ et l'on a évidemment

$$
\left|\varphi_{m}(t)\right| \leq g(t), \quad 0 \leq t \leq t_{m} .
$$

De l'inégalité (20) on déduit

$$
\int_{0}^{t_{m}} k\left(t_{m}, s\right) \varphi_{m}(s) d s>m G\left(t_{m}\right), \quad \quad m \geq 1 .
$$

D'après le théorème de Lusin concernant la structure des fonctions mesurables, on peut trouver une fonction continue $f_{m}(t)$, définie sur $0 \leq t \leq t_{m}$, telle qu' on ait

$$
\begin{array}{cr}
\left|f_{m}(t)\right| \leq g(t), & 0 \leq t \leq t_{m}, \\
\int_{0}^{t_{m}} k\left(t_{m}, s\right) f_{m}(s) d s>m G\left(t_{m}\right), & m \geq 1 .
\end{array}
$$

Les fonctions $\varphi_{m}(t)$ et $f_{m}(t)$ vont différer sur un ensemble de mesure (LEBESGtre) suffisamment petite pour assurer la condition (25), compte tenant de la condition (23).

Nous pouvons prolonger les fonctions $f_{m}(t)$ sur le demi-axe $R_{+}$, de telle manière que chacune soit continue et satisfasse à la limitation

$$
\left|f_{m}(t)\right| \leq g(t), \quad t \in R_{+} .
$$


O. Conduneanu: Problèmes globaux dans le théorie des équations, etc. 357

Si l'on aurait $T C_{g} \subset C_{G}$, d'après le lemme plus haut établi, il faudrait avoir $\left\|I f_{m}(t)\right\| \leqslant A G(t), t \in R_{+}$, où $A$ est une constante. Cela résulte du fait que la suite $\left\{f_{m}(t)\right.$ \} est bornée dans l'espace $O_{g}$. Or, les inégalités (25) nous montrent qu'une telle constante ne peut exister. Par conséquent, la nécessité de la condition (19) est établie dans le cas $n=1$.

Soit maintenant $n>1$. On peut écrire $k(t, s)$ sous la forme

$$
k(t, s)=\sum_{i, j=1}^{n} k_{i j}(t, s),
$$

où $k_{i j}(t, s)$ est un noyau matriciel dont tous les éléments sont nuls, à l'exception de l'éément qui occupe la position $(i, j)$. Si l'on désigne par $T_{i j}$ l'opérateur qui correspond à $k_{i j}(t, s)$, on peut juger sur lui de la même manière que dans le cas scalaire. On obtient ainsi une relation de la forme (19) pour chaque $k_{i j}(t, s)$, ce qui nous conduit immédiatement au résultat, en tenant compte de la relation (27).

Remarque. - On connaît plusieurs cas particuliers du théorême 3. Par exemple, le cas $g(t) \equiv G(t) \equiv 1$ est dù à V.R.VInokourov [15] qui a été amené à ce résultat en étudiant la stabilité des solutions des équations de Volmerra. Le cas $g(t)=\exp \{\alpha t\}, G(t)=\exp \{\beta t\}$ est dû à E. I. GoLdenhersheL [5] qui s'est occupé de la théorie spectrale des opérateurs de Volmerra. Mentionnons encore le travail de YU. I. NEIMARK [10], dans lequel on traite un problème analogue.

6. Le théorème 3 nous permet d'appliquer les théorèmes généraux 1 et 2 pour obtenir des critériums effectifs d'existence des solutions de l'équation (E). Nous allons indiquer seulement quelques conséquences du théorème 1 , en laissant de côté les questions semblables concernant le théorème 2.

Supposons qu' on cherche des solutions bornées de l'équation $(E)$, c'està-dire, $D=C$. En tenant compte des théorèmes 1 et 3 , on déduit aisément le rósultat suivant:

Proposimion 1. - Considérons l'équation (E) et admettons les conditions suivantes:

a) il existe un nombre $A>0$ et une fonction $g(t)>0$, continue sur $R_{+}$, tels que

$$
\int_{0}^{t}\|k(t, s)\| g(s) d s \leq A, \quad t \in R_{+}
$$


b) $f(t, x)$ est une fonction vectorielle continue pour $t \in R_{+},\|x\| \leq p$, telle que

$$
\begin{gathered}
f(t, 0) \in C_{g}, \\
\|f(t, x)-f(t, y)\| \leq \lambda g(t)\|x-y\| ;
\end{gathered}
$$

c) $h(t) \in C$.

Alors, il existe une seule solution $x(t) \in C$ de l'équation $(E)$, telle que $\|x(t)\| \leq p$ pour $t \in R_{+}$, dés que $\|h(t)\|, \lambda$ et $|f(t, 0)|_{g}$ sont assez petits.

Pour la démonstration, il suffit de remarquer que la condition (28) est la condition d'admissibilité de la paire $\left(C_{G}, C\right)$ par rapporl à l'opérateur $T$, pendant que les conditions (29) et (30) ont pour conséquence le fait que l'opérateur $x(t) \rightarrow f(t, x(t))$ est un opérateur de $C$ à $C_{g}$. Il ne reste qu'à appliquer le théorème 1 , en prenant $B=C_{g}$ et $D=C$.

Cette proposition nous donne des conditions, assez simples et générales, assurant l'existence et l'unicité de la solution de l'équation $(E)$. Mentionnons quelques cas particuliers.

Coroltaine 1. - Supposons que les conditions suivantes sont satisfaites:

a) $\int_{0}^{t}\|k(t, s)\| d s \leq A, \quad t \in R_{+}$;

b) $f(t, x)$ est continue pour $t \in R_{+},\|x\| \leq \rho, f(t, 0)$ est bornée sur $R_{+}$et

$$
\|f(t, x)-f(t, y)\| \leq \lambda\|x-y\| ;
$$

o) $h(t)$ est continue et bornée sur $R_{+}$.

Alors il existe une seule solution bornée (sur $R_{+}$) de l'équation (E), dès que $\|h(t)\|, \lambda$ et $\|f(t, 0)\|$ sont assez petits.

En effet, il suffit de prendre $g(t) \equiv 1$ dans l'énoncé de la proposition 1.

Corollaire 2. - Admettons les conditions:

a) $\|k(t, s)\| \leq K, \quad 0 \leq s \leq t<+\infty, \int_{0}^{\infty} g(s) d s<+\infty$;

b) et c), les mêmes que dans la proposition 1 .

Alors, la conclusion de la proposition 1 est valable.

La condition a) du corollaire 2 a, évidemment, pour conséquence la condition (28). 
C. Corduneanu: Problèmes globaux dans le théorie des équations, ete. 359

Corollarre 3. - Supposons satisfaites les conditions suivantes:

a) $\|k(t, s)\| \leq K \exp \{-\alpha(t-s)\}, 0 \leq s \leq t<+\infty, \sup _{t \geq 0}\left\{\int_{t}^{t+1} g(s) d s\right\}<+\infty$, $K$ et a étant des nombres positifs;

b) et c) les mêmes que dans la proposition 1.

Alors, il existe une seule solution $x(t) \in C$ de l'équation $(E)$, telle que $\|x(t)\| \leq \rho$ pour $t \in R_{+}$, dès que $\|h(t)\|, \lambda$ et $|f(t, 0)|_{g}$ sont assez petits.

Il est aisé de voir que la condition a) de l'énoncé du corollaire 3 a pour conséquence l'inégalité (28), si l'on tient compte d' une inégalité établie dans [8].

Proposinton 2. - Considérons l'équation (E) et admettons les conditions suivantes:

a) $\|k(t, s)\| \leq K \exp \{-\alpha(t-s)\}, 0 \leq s \leq t<+\infty$, oì $K$ et a sont des nombres positifs;

b) $f(t, x)$ est continue pour $t \in R_{+},\|x\| \leq \rho, f(t, 0) \equiv 0$ et

$$
\|f(t, x)-f(t, y)\| \leq \lambda\|x-y\| ;
$$

c) $\|h(t)\| \leq H \exp \{-\beta t\}, t \in R_{+}$, oì $\beta(0<\beta<\alpha)$ et $H>0$ sont des constantes.

Alors, il existe une solution unique $x(t)$ de l'equation $(E)$, satisfaisant $\grave{a}$ la limitation

$$
\|x(t)\| \leq \rho \exp \{-\beta t\}, \quad t \in R_{+},
$$

dès que $\lambda$ et $H$ sont assez petits.

La condition a) entraîne évidemment l'inégalité

$$
\int_{0}^{t}\|k(t, s)\| \exp \left\{-\beta s \mid d s \leq K(\alpha-\beta)^{-1} \exp (-\beta t\}, t \in R_{+}\right.
$$

ce qui nous montre que la paire $\left(C_{g}, C_{g}\right)$, avec $g=\exp \{-\beta t\}$, est admissible par rapport à l'opérateur $T$ généré par $k(t, s)$. Donc, on peut appliquer le théorème 1. Il en résulte l'existence et l'unicité de la solution de l'équation $(E)$, satisfaisant à la limitation (32). En particulier, on aura $\lim _{t \rightarrow+\infty}\|x(t)\|=0$.

Il est aisé de voir que la proposition 3 généralise aux equations intégrales de Volmenra le théorème bien connu de Poincané-Liapounoff sur 
C. Condunanu: Problèmes globaux dans le théorie des équations, etc.

la stabilité asymptotique, théorème qui concerne les systèmes différentiels ordinaires de la forme

$$
\dot{x}=A x+f(t, x)
$$

A étant une matrice hurwitzienne. En effet, le système différentiel est équivalent à l'équation

$$
x(t)=X(t) x_{0}+\int_{0}^{t} X(t-s) f(s, x(s)) d s,
$$

ou $\dot{X}(l)=A X(t), X(0)=E$.

Remarquons encore que le cas des noyaux de translation $k(t, s)=k(t-s)$ a été considéé par J. A. NoHeL [12] qui a utitisé la méthode de comparaison (inégalités intégrales).

\%. Dans ce dernier paragraphe, nous allons brièvemeut indiquer un autre procédé qui permet d'obtenir des résultats à caractère global pour les équa. tions intégrales de Vocterra de la forme générale

$$
x(t)=h(t)+\int_{0}^{t} k(t, s, x(s)) d s .
$$

Ce procédé repose aussi sur le théorème du point fixe de SoHadder et TroHoNOFF.

Thtónteme 4. - Considérons l'équation $\left(E^{\prime}\right)$ et admettons les conditions suivantes:

a) la fonction vectorielle $k(t, s, x)$ est continue pour $0 \leq s \leq t+\infty$, $\|x\| \leq g(t)$, oì $g(t)$ est une fonction continue et positive sur $R_{+}$;

b) il existe une fonction $K(t, s)$, continue pour $0 \leq s \leq t<+\infty$, telle qu' on a $\|k(t, s, x)\| \leq K(t, s)$ pour $\|x\| \leq g(t)$;

c) $h(t) \in C_{c}\left(R_{+}, R^{n}\right)$;

d) les fonctions $h(t), K(t, s)$ et $g(t)$ satisfont à l'inégalité

$$
\|h(t)\|+\int_{0}^{t} K(t, s) d s \leq g(t), \quad t \in R_{+} .
$$

Alors, il existe au moins une solution $x(t)$ de l'équation $\left(E^{\prime}\right)$ définie pour $t \in R_{+}$et telle que

$$
\|x(t)\| \leq g(t), \quad t \in R_{+} .
$$


C. Corduneanu: Problèmes globaux dans le théorie des équations, etc. 361

Démonstration. Daus l'espace $C_{c}\left(R_{+}, R^{n}\right)$ on considère l'ensemble convexe et fermé $M=\left\{x(t) ;\|x(t)\| \leq g(t), t \in R_{+}\right\}$. Sur cet ensemble on définit l'opérateur continu

$$
V x(t)=h(t)+\int_{0}^{t} k(t, s, x(s)) d s .
$$

Il est aisé de voir que l'ensemble $A=\overline{V M}$ (la fermeture étant prise par rapport à $C_{e}\left(R_{+}, R^{n}\right)$ ) est compact dans $C_{e}\left(R_{+}, R^{n}\right)$. La condition (34) de l'énoncé assure l'inclusion $V M \subset M$. On aura done $V M \subset A \subset M$, ce qui nous permet de considérer le théorème 4 démontré.

L'application da théorème 4 revient, au fond, à la recherche des fonctions $K(t, s)$ et $g(t)$, une fois donnée l'équation $\left(E^{\prime}\right)$. Pour donner un exemple, supposons $q u^{\prime}$ on peut trouver une fonction (scalaire) continue $G(t, s, u)$, définie pour $0 \leq s \leq t<+\infty, 0 \leq u \leq g(t)$, telle qu' on ait

$$
\|k(t, s, x)\| \leq G(t, s,\|x\|)
$$

De plus, supposons que $G(t, s, u)$ est non décroissante par rapport à $u$. Alors on pent prendre $K(t, s)=G(t, s, g(s))$, et l'inégalité $(3 t)$ devient

$$
\|h(t)\|+\int_{0}^{t} G(t, s, g(s)) d s \leq g(t) .
$$

Il en résulte que la fonction $g(t)$ doit être cherchée parmi les solutions de l'inégalité intégrale (38). En particulier, si l'équation intégrale scalaire qu' on peut associer à (38)

$$
g(t)=\|h(t)\|+\int_{0}^{t} G(t, s, g(s)) d s
$$

admet une solution $g(t)$ définie sur $R_{+}$, alors l'équation $\left(E^{\prime}\right)$ admet aussi une solution définie sur $R_{+}$, satisfaisant à la limitation (35).

Il en résulte que les inégalités intégrales de la forme (39) sont étroitement liées au problème du comportement global des solutions des équations intégrales non linéaires de VoLTerRa. C'est d'ailleurs un fait mis en évidence par plusieurs auteurs [13], [11], [1].

Considérons de nouveau l'équation $(E)$ et supposons qu'il existe une fonction $F(t, u)$, continue pour $0 \leq t<+\infty, 0 \leq u \leq g(t)$, telle qu' on a

$$
\|f(t, x)\| \leq F(t,\|x\|) .
$$


Si, de plus, $F(t, u)$ est non décroissante par rapport à $u$, on peut prendre alors $K(t, s)=\|h(t, s)\| F(s, g(s))$. L' équation (scalaire) de comparaison aura done la forme

$$
g(t)=\|h(t)\|+\int_{0}^{t}\|k(t, s)\| F(s, g(s)) d s .
$$

La manière dont on peut atiliser l'équation (41), dans l'etude des propriétés globales des solutions de l'équation $(E)$, est indiquée dans le travail de A. STOKBs [14]. Ce travail est consacré à l'étude des systémes différentiels de la forme

$$
\dot{x}=A(t) x+f(t, x),
$$

mais il n'y a aucune difficulté quand on passe aux équations intégrales de la forme $(E)$. Vu que tout système de la forme $(S)$ est équivalent à une équation intégrale vectorielle de la forme $(E)$, on peut obtenir de cette façon des résultats qui généralisent les résultats établis dans [14].

\section{BIBLIOGRAPHIE}

[1] Azbertiv N. V., Tzaliuk Z. B., Sur les inégalités intégrales I. (en russe). Mat. Sbornik, t. 56 (1962), p. 325-342.

[2] Brelecki A-, Une remarque sur la méthode de Banach-Caccioppoli-Tikhonov. Bull. Acad. Polonaise Sci., t. IV (1956), p. 255-261.

[3] Bourbakr N., Topologie générale, Oh. IX, X, Paris, 1948-1949.

[4] Conduneand C., Sur certains systèmes différentiels non-linéaires Analele st. Univ. Iasi, t. IV (1960), p. $257-260$.

[5] GoLdenHershel E. I., Le spectre de l'opérateur de Volterra sur un demi-axe et la croissance exponentielle des solutions des systèmes des équations intégrales de Volterra (en russe). Mat. Sbornik, t. 64 (1964), p. 110̃-139.

[6] Hartman Ph., Onuchio $\mathrm{N}$, On the asymptotic integration of ordinary differential equations. Pacific Journal of Math. t. 13 (1968), p. 1198-1207.

[7] HILLE E., Functional analysis and semi-groups, Providence, 1957.

[8] MASSERA J. L., SOHÄFFER J. J., Linear differential equations and functional analysis I. Annals of Math. t. 67 (1958), p 517-573.

[9] - - Linear differential equations and functional analysis. Math. Annalen, t. 139 (1960), p. 287-342.

[10] Nămank Yu. I., Sur la permission de la linéarisation dans l'étude de la stabilité (en russe). Doklady AN SSSR, t. 127 (1959), p. 961-964.

[11] Noнec J. A., Some problems in nonlinear Volterra equations. Bull. Amer. Math. Soc, t. 10 (1962), p. 323-329. 
C. Condunganu: Problèmes globaux dans le théorie des équations, etc. 363

[12] - - Problems in qualitative behavior of solutions of nonlinear Volterra equations. Nonlinear integral equations. University of Wisconsin Press, Madison, 1964.

[13] Satō T., Sur l'équation intégrale non-linéaire de Volterra. Proc. Japan. Acad., t. 11 (1953), p. 271-290.

[14] STokes A., The applications of o fixed point theorem to a variety of non-linear stabi. lity problems. Contributions to differential equations V. Princeton Univ. Press, Prin. ceton 1960.

[15] Vrмокоurov V. R., Sur la stabilité des solutions des systèmes a'équations intégrales de Volterra de la seconde espèce (en russe). Izvestja VUZ, Matematika (1959), No. 1 (8), p. 23-34. 